\title{
Neurobiological and Endocrine Correlates of Individual Differences in Spatial Learning Ability
}

\author{
Carmen Sandi, ${ }^{1,2,4}$ M. Isabel Cordero, ${ }^{1,2}$ José J. Merino, ${ }^{1}$ Nyika D. Kruyt, ${ }^{1}$ \\ Ciaran M. Regan, ${ }^{3}$ and Keith J. Murphy ${ }^{3}$ \\ ${ }^{1}$ Department of Psychobiology, Universidad Nacional de Educacion a Distancia, Ciudad Universitaria s/n, 28040 Madrid, Spain; \\ ${ }^{2}$ Brain and Mind Institute, EPFL, CH-1015 Lausanne, Switzerland; ${ }^{3}$ Department of Pharmacology, Centre for Integrative Biology, \\ The Conway Institute, University College, Belfield, Dublin 4, Ireland
}

\begin{abstract}
The polysialylated neural cell adhesion molecule (PSA-NCAM) has been implicated in activity-dependent synaptic remodeling and memory formation. Here, we questioned whether training-induced modulation of PSA-NCAM expression might be related to individual differences in spatial learning abilities. At $12 \mathrm{~h}$ posttraining, immunohistochemical analyses revealed a learning-induced up-regulation of PSA-NCAM in the hippocampal dentate gyrus that was related to the spatial learning abilities displayed by rats during training. Specifically, a positive correlation was found between latency to find the platform and subsequent activated PSA levels, indicating that greater induction of polysialylation was observed in rats with the slower acquisition curve. At posttraining times when no learning-associated activation of PSA was observed, no such correlation was found. Further experiments revealed that performance in the massed water maze training is related to a pattern of spatial learning and memory abilities, and to learning-related glucocorticoid responsiveness. Taken together, our findings suggest that the learning-related neural circuits of fast learners are better suited to solving the water maze task than those of slow learners, the latter relying more on structural reorganization to form memory, rather than the relatively economic mechanism of altering synaptic efficacy that is likely used by the former.
\end{abstract}

Although the existence of variation in learning abilities among individuals belonging to the same species has been recognized for a long time (James 1890; Habib et al. 2000; Nguyen et al. 2000), the neurobiological correlates underlying such variability are largely unknown. In humans, imaging studies have shown different patterns of brain activation to be associated with different levels of learning and memory performance (Habib et al. 2000; Cazalis et al. 2003). Moreover, activity within the hippocampus during encoding has also been shown to correlate, across individuals, with performance on tests of memory tasks (for review, see Tulving et al. 1999). Evidence indicates that rats can considerably differ in their spatial learning abilities, a phenomenon particularly prominent at senescence (Rowe et al. 1998; Schulz et al. 2002), but also observable in early adulthood (Adams et al. 2001; Sandi et al. 2003a; Venero et al. 2004). Recent reports support the view that individual differences in the acquisition of the hippocampus-dependent learning task the Morris water maze (Morris et al. 1982; Riedel et al. 1999) are associated with a differential modulation of specific hippocampal circuits. Both in young and aged rats, the abundance of the NR1 subunit of the NMDA receptor in the dendrites of CA3 pyramidal cells was reported to correlate with performance variability during maze learning, with animals that performed better exhibiting higher NR1 levels (Adams et al. 2001). Moreover, we recently found the inverse correlation between learning ability and synaptic density at the level of the contacts between mossy fiber terminals and proximal apical dendrites from CA3 pyramidal cells observed $24 \mathrm{~h}$ following a massed training protocol, with

\footnotetext{
${ }^{4}$ Corresponding author.

E-MAIL carmen.sandi@epfl.ch; FAX 41-21-693-9636.

Article and publication are at http://www.learnmem.org/cgi/doi/10.1101/ $\operatorname{lm} .73904$.
}

synaptic levels being higher in rats that exhibited a poorer acquisition curve (Sandi et al. 2003a). Such a finding suggested that greater neural remodeling-in terms of new synapse formationoccurs in animals that require a greater effort to learn the task. It could, therefore, be hypothesized that the remodeling of hippocampal circuits underlying the processing of spatial information presents differential characteristics depending on the performance rate displayed by rats during acquisition of the task.

In this study, we aimed to assess whether polysialylation of the neural cell adhesion molecule (NCAM) in the hippocampus might also show a differential learning-induced regulation depending on rats' performance at training. NCAM is a member of the immunoglobulin superfamily of adhesion molecules which, through homophilic and heterophilic binding, plays key roles in morphogenesis and synaptic plasticity (Schachner 1997; Rønn et al. 2000). NCAM can be polysialylated by the attachment of long chains of $\alpha 2,8$-linked polysialic acid (PSA) homopolymers. Such a posttranslational modification is believed to inhibit NCAMmediated cell-cell interactions (Sadoul et al. 1983; Rougon 1993; but see also Durbec and Cremer 2001) and has been reported to play key roles in activity-dependent synaptic remodeling (Nothias et al. 1997; Kiss 1998; Theodosis et al. 1999; Kiss and Muller 2001) and memory storage (Murphy and Regan 1998). In particular, the frequency of NCAM polysialylated neurons at the infragranular border of the hippocampal dentate gyrus has been shown to present transient increases $10-12 \mathrm{~h}$ after training rats in a variety of learning tasks, including the Morris water maze (Doyle et al. 1992; Fox et al. 1995; Murphy et al. 1996; Foley et al. 2003; Sandi et al. 2003b). The timing of this transient increase in PSA-NCAM expression may play a role in hippocampal synapse selection, a process thought to be ongoing during this time period (O'Malley et al. 2000). Moreover, specific removal of PSA on NCAM with the enzyme endoneuraminidase (EndoN) impairs activity-induced synaptic potentiation (Becker et al. 1996; Muller 
et al. 1996) and spatial memory (Becker et al. 1996). Interestingly, PSA-NCAM is expressed in the mossy fiber axons of dentate granule cells (Seki and Arai 1991, 1999), which establish multiple asymmetrical synapses with the most proximal portion of the apical dendrites of CA3 pyramidal neurons.

A second aim of this study was to further characterize the implications, at the behavioral and endocrine levels, of individual variation observed in the acquisition of the massed water maze protocol. Thus, we questioned whether good learners identified with such a procedure would maintain their better performance skills if submitted to subsequent learning and memory tests, and whether the opposite would be the case for poor learners. Furthermore, because learning and memory processes in this task have been shown to depend on the hippocampal action of glucocorticoid hormones (Oitzl and de Kloet 1992; Roozendaal and McGaugh 1997; Sandi et al. 1997), we also explored whether differences in performance in such a task would be coupled to differences in corticosterone levels assayed after submitting rats to the different testing protocols.

\section{RESULTS}

\section{Relationship Between Water Maze Learning} and the Frequency of PSA-Immunoreactive Neurons in the Hippocampal Dentate Gyrus

Experiment 1 investigated whether spatial learning abilities could be related to a subsequent differential modulation of PSANCAM expression in the dentate gyrus. As shown in Figure 1A, rats exhibited progressively shorter latencies to find the platform throughout the trials of the massed training procedure used in this study. In agreement with previous findings (Venero et al. 2004), we observed a significant positive correlation between the mean latency to finding the platform during the training session (trials 2-8) and thigmotactic swimming behavior $(r=0.86$, $P<0.001)$; that is, the slower the animals to find the platform, the higher their levels of thigmotactic behavior. Furthermore, acquisition of the task was also evinced by the significant amount of time they used searching for the platform in the target quadrant during the probe test given $12 \mathrm{~h}$ later $(34.4 \% \pm 2 \%$; significantly above chance performance, 25\%). Correlational analysis between the mean latency to reach the platform at train- ing and the percent time spent in the target quadrant during the probe test showed a trend toward a negative correlation $(r=-0.48, p=0.12$; n.s.).

As previously described (Fox et al. 1995), PSA immunohistochemistry revealed a discrete group of neurons at the infragranular zone of the hippocampal dentate gyrus showing NCAM polysialylation (Fig. 2). Immunoreactivity was observed on the cell surface and dendrites that extended through the granule cell layer and into the molecular layer, as well as on the mossy fibers of these immunopositive cells. As shown in Figure 1B, and in agreement with previous findings (Murphy et al 1996; Sandi et al. 2003b), water maze learning significantly increased the number of polysialylated neurons observed in this brain region, $12 \mathrm{~h}$ following training, as compared with control rats $(t=2.8$, $\mathrm{df}=19, p<0.02)$.

Correlation analyses were performed between the latency required by rats to find the hidden platform during the training session (average latency for trials 1-8) and the number of polysialylated neurons quantified in the infragranular zone of the dentate gyrus at $12 \mathrm{~h}$ posttraining. As can be seen in Figure $1 \mathrm{C}$, a positive correlation was observed between these two parameters $(r=0.67, p<0.02)$, indicating that the poorer the performance in the water maze, the higher the number of PSA-immunopositive cells, and vice versa. Furthermore, we reanalyzed our previously published data, in which a time-dependent modulation of infragranular PSA-immunoreactive cells was described after training rats in a five-trial water maze session (Fig. 3A; Murphy et al. 1996). As can be seen in Figure 3A, PSA expression showed a tendency to increase at $10 \mathrm{~h}$ posttraining and was significantly increased at the $12 \mathrm{~h}$ posttraining time point. However, no such activation was observed at any of the other time points examined $(0,6,14$, and $24 \mathrm{~h}$ posttraining). Interestingly, the same positive correlation as described above was also found at the 12 -h posttraining time point $(r=0.85, p<0.03$; Fig. 3B). However, no such correlation existed at any other posttraining time examined, times at which no learning-associated activation of polysialylation state was observed (Fig. 3B). In addition, no such correlation was evident in passive or visuomotor control groups that were allowed to swim for an equivalent time to trained counterparts in the absence of a platform or trained with a visible platform above the surface of the water, nor in animals rendered amnesic for the task by scopolamine injections (Fig. 3B). It was noteworthy that no qualitative difference in background PSA levels or the inten-
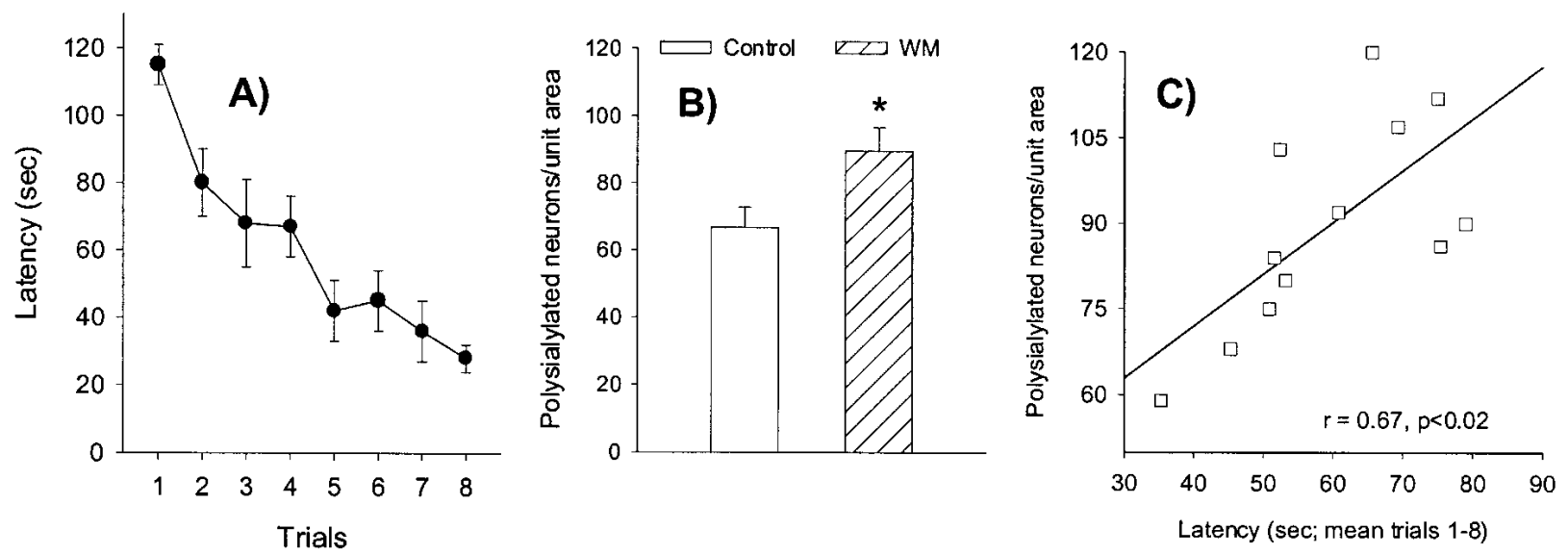

Figure 1 Influence of water maze training on frequency of dentate polysialylated neurons at $12 \mathrm{~h}$ posttraining. $(A)$ The acquisition curve displayed by rats to learn the water maze learning, with latency decrease with increasing trials to find the platform. $(B)$ The increase in polysialylated neurons in the infragranular cell layer of the dentate gyrus observed $12 \mathrm{~h}$ posttraining. Data represent mean \pm SEM (control group, $n=9$; water maze [WM], $n=12$ ); $\left.{ }^{*}\right) p<0.05$ versus control group. (C) The correlation between the learning performance in the water maze (latency to find the platform) and the frequency of dentate polysialylated neurons at $12 \mathrm{~h}$ posttraining. 

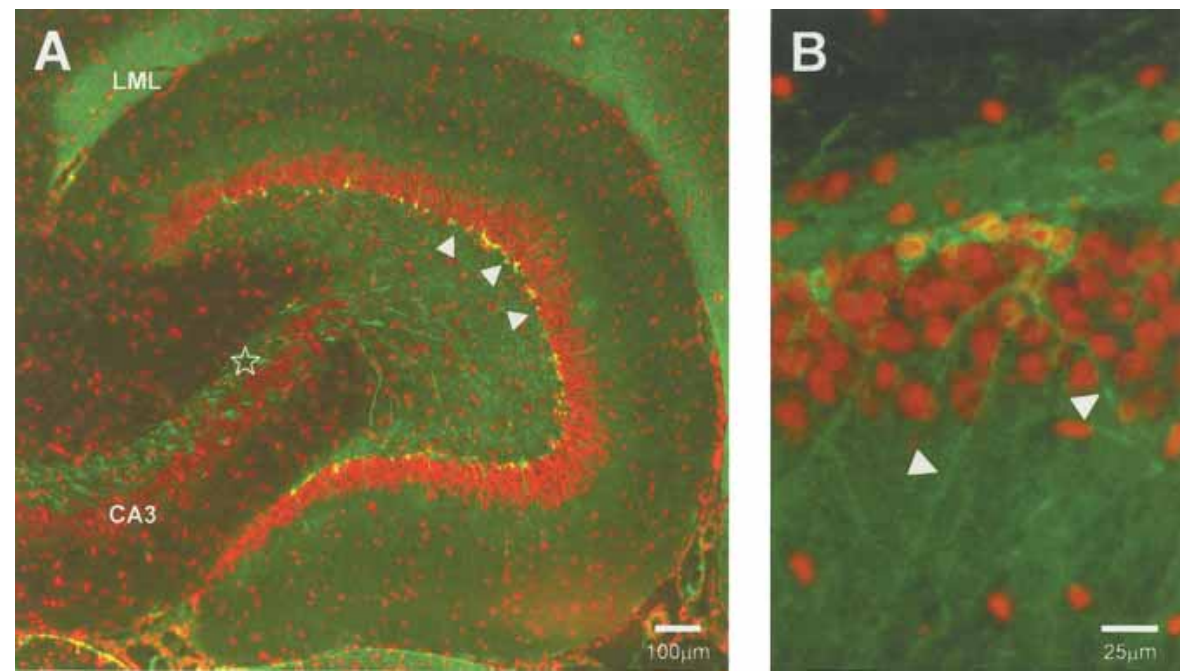

Figure 2 Photomicrographs of dentate polysialylated neurons in the hippocampal formation. $(A)$ Intense labeling of the polysialylated neurons at the infragranular zone of the hippocampal dentate gyrus (arrowheads) and mossy fiber bundle (star). (B) A higher magnification of the dentate polysialylated neurons. The PSA immunoreactivity is clearly localized to the cell body membrane and their dendritic processes (arrowheads) that extend through the granule cell layer into the dentate molecular layer. Both sections are counterstained with the nuclear marker propidium iodide (red) to allow identification of cell bodies. (LML) Lacunar molecular layer.

sity of PSA immunofluorescence on infragranular neurons was evident in the dentate gyrus of slow compared with fast learners.

\section{Relationship Between Individual Variation in the} Acquisition of a Massed Water Maze Training Protocol and Subsequent Behavioral and Endocrine Responses

Given that we found a relationship between learning abilities in a massed water maze training protocol and (1) PSA-NCAM regulation in cells of the dentate gyrus at $12 \mathrm{~h}$ posttraining (Experiment 1 ), and (2) synaptic density between mossy fibers and proximal apical dendrites of CA3 neurons at $24 \mathrm{~h}$ posttraining (Sandi et al. 2003a), a second aim of this study was to explore to what extent individual variation in performance detected with our learning task was indicative of a consistent pattern of spatial learning abilities. In other words, would there be a relationship between performance of rats in this protocol and their performance when subsequently confronted with other learning and memory challenges in the same maze? Moreover, would such performance variability be related to differences in corticosteroid activation?

To address these issues, we set up three experiments, in which rats trained in the massed water maze training protocol on day 1, were then submitted on day 2 to (1) a probe test (Experiment 2); (2) a relearning session (Experiment 3); or (3) a reversal learning session (Experiment 4).

As shown in Figure 4A (Experiment 2), a negative correlation was found between the mean latency to reach the platform in the massed training protocol and the percent time spent in the "target" quadrant (where the platform was located at training) during the first $30 \mathrm{sec}$ of the probe test $(r=-0.83, p<0.001)$. Thus, the lower the time required to acquire the spatial learning task on day 1, the longer the time spent in the target quadrant on day 2 , and vice versa. Hence, these results indicated a relationship between performance at learning and the level of retention of the task at testing. However, when the $60 \mathrm{sec}$ of the probe test was considered, the negative relation between these two variables only approached significance $(r=-0.51, p<0.09)$.

As can be observed in Figure 4B (Experiment 3), a positive correlation was found between the mean latency to reach the platform in the massed training protocol and the latency displayed by animals during the relearning session $(r=0.68, p<0.02)$. Therefore, the level of performance exhibited during the first acquisition test was in concordance with subsequent performance under the same spatial demands. Moreover, as shown in Experiment 4 (Fig. 4C), a positive correlation was also found between the mean latency to reach the platform in the massed training protocol and the latency displayed by animals during the reversal learning session $(r=0.83$, $p<0.03)$. According to this correlation, the lower the time required to acquire the spatial learning task on day 1 , the lower the time required to learn a new spatial location in day 2 , and vice versa. Hence, this indicates a good concordance between performance abilities in the two spatial tasks.

Plasma corticosterone levels were evaluated in all groups of animals immediately after behavioral testing on day 2 , and were compared with a group of undisturbed rats $(n=9 ; 63.3 \pm 10.5 \mathrm{ng} / \mathrm{mL})$. In Experiment 2, values from rats submitted to a 60 -sec probe test in day 2 $(71.1 \pm 13.6 \mathrm{ng} / \mathrm{mL}$ ) did not differ from those of controls (n.s.), and no correlation was found with performance in the water maze on either testing day. In Experiment 3, water-maze-trained rats showed a significant enhancement of corticosterone levels after relearning $(488.1 \pm 58.3 ; p<0.001)$ as compared with undisturbed rats. In these animals, a positive correlation was found between corticosterone levels after training on day 2, and mean latency to find the platform both during acquisition of the task on day $1(r=0.68, p<0.03$; Fig. $5 \mathrm{~A})$ and during relearning on day $2(r=0.67, p<0.03$; Fig. 5B). Therefore, the lower the latencies to find the platform in both sessions, the lower the plasma corticosterone levels. Finally, as compared with undisturbed controls, corticosterone levels were also found to be significantly enhanced in rats submitted to reversal learning in day 2 $(654.6 \pm 84.3 ; p<0.001)$. However, no significant correlations were found in these rats between hormone levels and behavioral parameters.

\section{DISCUSSION}

In agreement with previous findings (Murphy et al. 1996), here we show that rats trained in a one-session massed water maze exhibited a significant increase in dentate polysialylation $12 \mathrm{~h}$ posttraining. We also show, for the first time, that such traininginduced up-regulation of NCAM polysialylation is related to the spatial learning abilities displayed by rats during training. Specifically, a positive correlation was found between mean latency to learn the platform location at training and the activated frequency of dentate polysialylated neurons, indicating that the higher polysialylation response was observed in rats showing the slower acquisition rate. Interestingly, no such correlation existed at any other time point examined $(0,6,14$, and $24 \mathrm{~h}$ posttraining), times at which no learning-associated activation of polysialylation state was observed.

These results further support our hypothesis put forward above that neural circuits subserving learning in fast and slow

\section{Learning \& Memory}


A

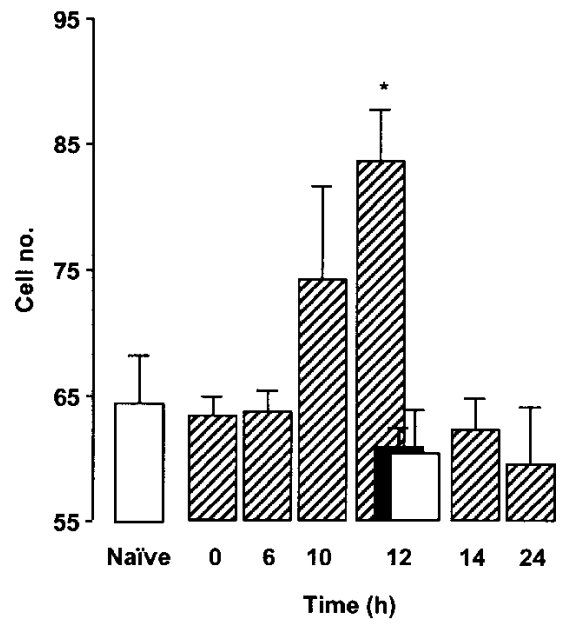

B

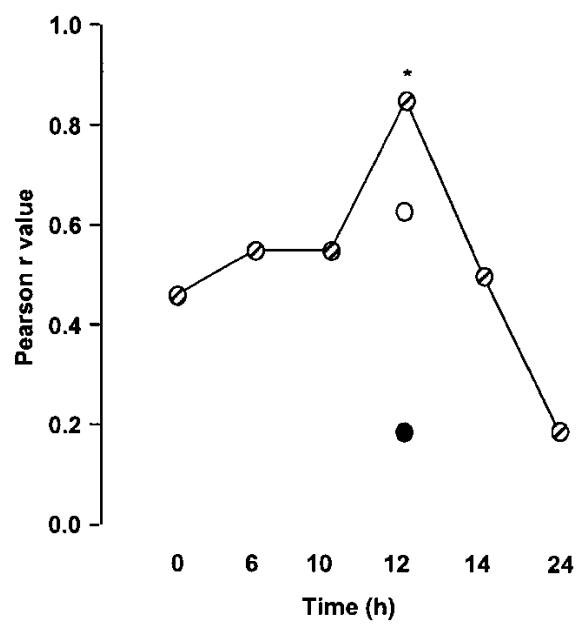

Figure 3 The relationship between previous learning in the water maze and frequency of hippocampal infragranular PSA-immunoreactive cells at increasing times posttraining. (A) Frequency of PSA-immunoreactive cells in the granule cell layer/hilar border region of the rat hippocampal dentate gyrus at increasing posttraining times (adapted from Murphy et al. 1996). Values are the mean \pm SEM number of cells $/ 0.15 \mathrm{~mm}^{2}$ of the granule cell layer $(4 \leq n \leq 6)$. Those significantly different $(p<0.05)$ from naive are indicated with an asterisk. (B) Pearson correlation $r$-values for comparison of PSApositive cell frequency to the average escape latency across all five trials for each time point following training ( $4 \leq n \leq 6)$. Significant correlation is indicated by an asterisk. In both panels, passive animal controls, animals allowed to swim in the pool in the absence of a platform for a period of time matching their trained counterparts, are indicated by the white column/circle. Animals rendered amnesic by scopolamine ( $0.8 \mathrm{mg} / \mathrm{kg}$, i.p.; $20 \mathrm{~min}$ prior to training) are indicated by the black column/ circle.

learners show a differential training-induced regulation of synaptic remodeling mechanisms. PSA activation has been proposed to participate in learning-associated synaptic remodeling (Doyle et al. 1992; Murphy and Regan 1998). Using the same massed training protocol as was used in the current study, we recently found a learning-related differential expression of asymmetric synapses between mossy fiber boutons and CA3c pyramidal cells $24 \mathrm{~h}$ post-training. Synaptic density was shown to be higher in animals that showed a poorer acquisition curve (Sandi et al. 2003a). These findings, together with the present correlation between difficulty of task acquisition and activation of PSA-NCAM expression, strongly suggest that a greater structural reorganization of neural circuits occurs in the hippocampus of animals that require a greater effort to learn the task.

Hippocampal PSA has been related to bouton formation and remodeling that accompany synapse formation between certain types of mossy fiber boutons and CA3 pyramidal cells (Seki and Arai 1999). Such a link between PSA expression and synaptic modulation in CA3 is further supported by studies showing that removal of PSA by specific enzymatic degradation or mutation of the NCAM-180 isoform induces aberrant innervation of the CA3 pyramidal cells by mossy fibers (Seki and Rutishauser 1998). Thus, as modulated PSA-NCAM expression is carried on the same mossy fiber axonal projections that are associated with increased synaptic contacts in slow learners, it may well be that increased PSA expression directly facilitates such effortful learningassociated synaptic reorganization. Moreover, given reports of synaptic reorganization at perforant path/granule cell synapses following water maze training ( $\mathrm{O}^{\prime}$ Malley et al. 2000; Eyre et al. 2003), our findings indicate that memory consolidation is accompanied by structural remodeling at both the dendritic and axonal synaptic fields of the hippocampal dentate granule cells. Whether learning-associated reorganization of perforant path/ granule cell synapses is similarly seen predominantly in slow learners remains to be established; however, as activated PSA-
NCAM is also expressed on the granule cell dendritic trees receiving entorhinal input, this seems likely.

Although the evidence just reviewed supports a role of PSA in individual variability in the encoding of the water maze experience, alternative interpretations should also be considered. Thus, it could be hypothesized that differences in basal levels of PSA expression between fast and slow learners could account for their different performance in the spatial orientation task. However, such a proposal is not sustained owing to the lack of correlation observed immediately after training ( 0 -h time point). It could also be hypothesized that PSA expression is related to the differential activation of stress hormones here reported. However, the available evidence does not support this hypothesis, because increased levels of corticosterone have been related to decreased levels of PSA in the hippocampus (Rodriguez et al. 1998; Merino et al. 2000), and in our study the higher PSA levels were found in rats displaying the higher corticosterone levels (i.e., the slow learners), indicating that other factors should account for the observed modulation of PSA. Nevertheless, the possibility that the greater effort made by slow learners to learn the task could lead to a higher/stronger recruitment of neuronal circuits, which might, in turn, induce higher PSA expression, cannot be discarded. Interestingly, a recent report has presented evidence for a similar correlation between proficiency to learn a lever-press task and the expression of activity-regulated cytoskeleton-associated protein (Arc) mRNA in several brain regions-including the hippocampus-that is, animals that were slower to acquire the task exhibited higher levels of Arc mRNA than more proficient animals during the first acquisition session, but not at later stages of learning (Kelly and Deadwyler 2003).

Increasing evidence suggests that changes in factors that affect hippocampal circuitry might also explain the more pronounced variability in spatial learning ability observed in aged subjects. Individual differences in spatial learning capacity among aged rats have been shown to correlate with a variety of parameters in specific hippocampal subregions. For example, in comparison with animals displaying spatial memory impairments (i.e., the aged-impaired rats), animals with preserved spatial memory (i.e., the aged-unimpaired rats) exhibited (1) a higher level of cell proliferation and a higher number of new neurons (Drapeau et al. 2003); and (2) a higher expression of the presynaptic vesicle glycoprotein, synaptophysin (Smith et al. 2000). However, PSA expression was not found to differ between aged-unimpaired and aged-impaired rats (Abrous et al. 1997), although the fact that the only available study was performed several days posttraining does not preclude the possibility that, as in our work, a differential modulation could be observed in the immediate posttraining period (e.g., $10-12 \mathrm{~h}$ after the first training session).

Previously, it has been suggested that a significant proportion of the polysialylated dentate neurons are de novo granule cell precursors (Seki and Arai 1993). However, previous immunohistochemical detection of dentate cells with incorporated BrdU clearly demonstrated that a negligible number of PSA-positive 

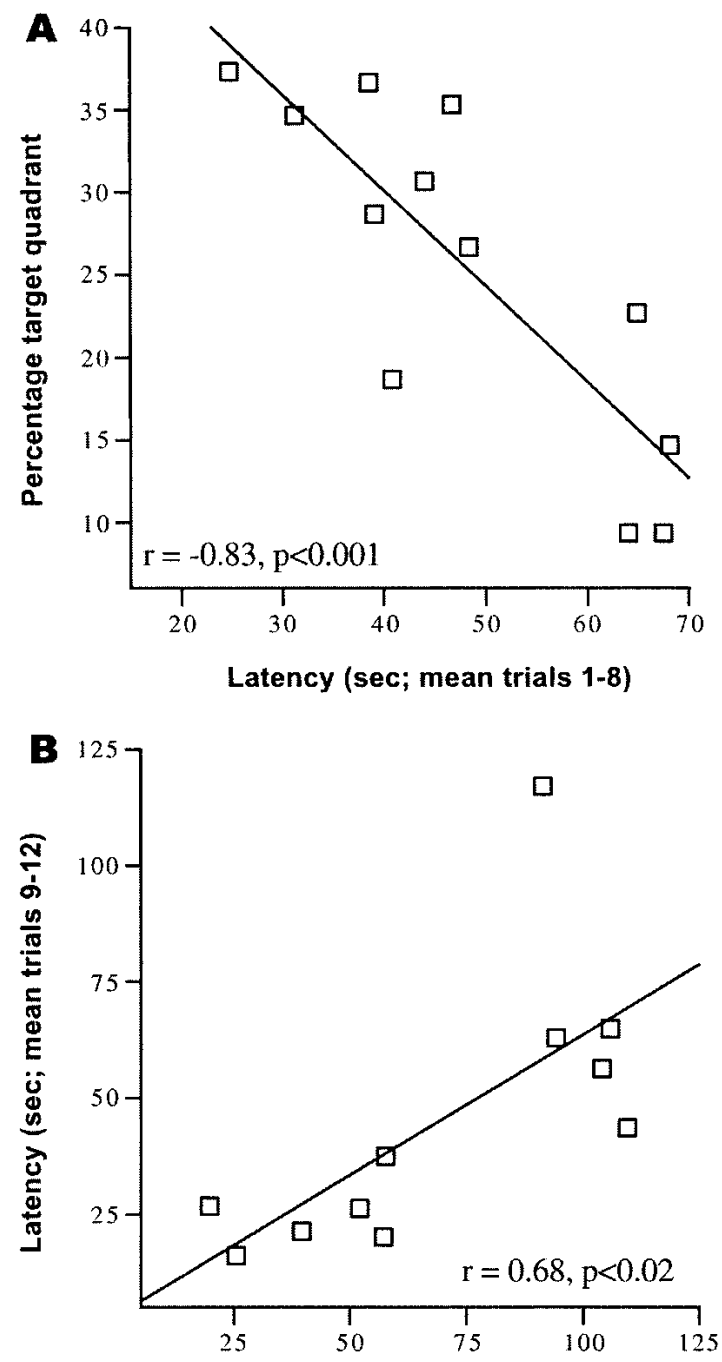

Latency (sec; mean trials 1-8)

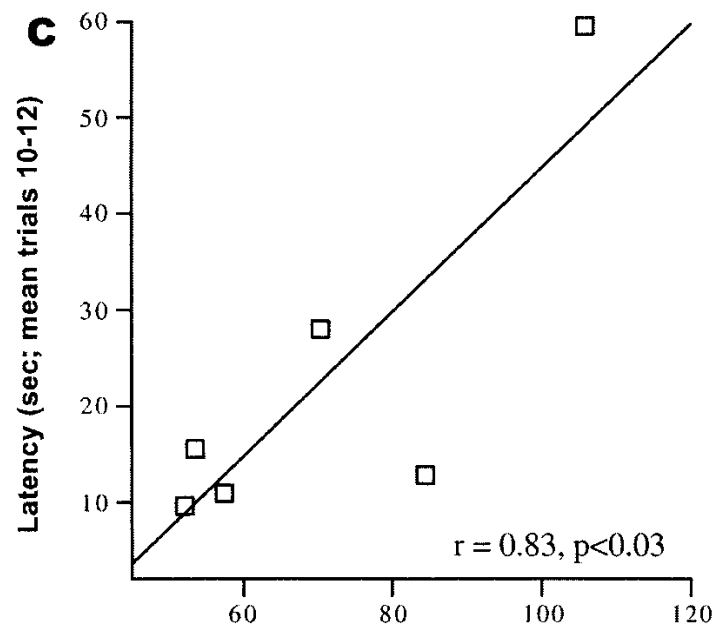

Latency (sec; mean trials 1-8) cells observed $12 \mathrm{~h}$ following learning were newly generated (Fox et al. 1995). Furthermore, evidence indicates that PSA-NCAM is not only expressed by newly born cells, but also by other cell types (Rodriguez et al. 1998; Banasr et al. 2001). This negates the possibility that the difference in PSA expression observed between the fast and slow learners reflects differential neurogenic rates. Nevertheless, it should be noted that differential neurogenesis seems to be associated with variability in water maze performance in aged, but not young adult rats (Drapeau et al. 2003).

The question arises, then, as to what extent could our findings on hippocampal up-regulation of PSA-NCAM and synapse formation be related to spatial memory formation, if they are specifically observed in the "slow" but not the "fast" learners? A first important point to be considered is that, although at a different rate and to a different degree, all animals learned the task. For example, all animals trained in the massed trial protocol in Experiment 1 found the platform in at least three out of the last four trials (trials $5-8$ ) in $<50 \mathrm{sec}$, with their latency in at least one of these trials being $<30 \mathrm{sec}$. Furthermore, if classified into two subgroups with a "fast" learners group containing all the animals with a mean latency score below the mean, and the "slow" learners group the remainder, "fast" learners invariably find the platform on trial 2, in an average latency of $50 \mathrm{sec}$, as opposed to "slow" learners, $80 \%-100 \%$ of whom do not find the platform during the $120 \mathrm{sec}$ of trial 2 (data not shown). Because trial 2 is the first time rats are confronted with the task of finding the platform the location of which they were shown in trial 1, these observations reveal that fast learners use a more efficient problem-solving strategy than slow learners from the very beginning of the training procedure. In fact, slow learners showed high levels of thigmotactic swimming, which is characterized by navigating in circles and interferes with the development of an active searching strategy to find the escaping platform. Interestingly, such a thigmotactic behavior could reflect high anxiety levels in slow learners (Simon et al. 1994), which, in turn, might interfere with an effective task acquisition (McNaughton 1997). It is also interesting to note that a lack of correlation between PSA cell number and swim time was observed in passive controls (which swim for the same time as trained rats) and in animals rendered amnesic by scopolamine injections, at the 12-h time point (Fig. 3). Furthermore, the functional relevance of PSA regulation for memory processes has been provided by studies in which the enzymatic removal of PSA with endoneuraminidase-N impaired water maze performance (Becker et al. 1996) and hippocampal long-term potentiation (LTP; Becker et al. 1996; Muller et al. 1996). Thus, it might be reasoned that PSA-NCAM-mediated synaptic reorganization in the dentate gyrus is particularly involved in the processing/storage of "challenging" information, a situation in the water maze that would correspond with the behavioral demands impinging on slow learners.

The question remains as to what alternative mechanisms are used by the fast learners to facilitate such efficient storage of spatial information in the water maze task? In rats, individual differences in learning a spaced water maze protocol were found

Figure 4 Correlations between learning performance in a massed water maze training session on day 1 , and subsequent learning abilities in a variety of tasks involving different challenges in the same maze on day 2 . (A) The negative correlation found between average latency to platform during training (trials 1-8) and the percent time spent in the target quadrant during the transfer test performed on day $2(n=12$; Experiment 2). (B) The positive correlation observed between average latency to platform displayed at training (trials $1-8$, day 1 ) and at relearning (trials 9-12, day 2; $n=11$; Experiment 3). (C) The positive correlation found between average latency to platform displayed at training (trials $1-8$, day 1 ) and at reversal learning (trials $10-12$, day 2; $n=6$; Experiment 4). 
A
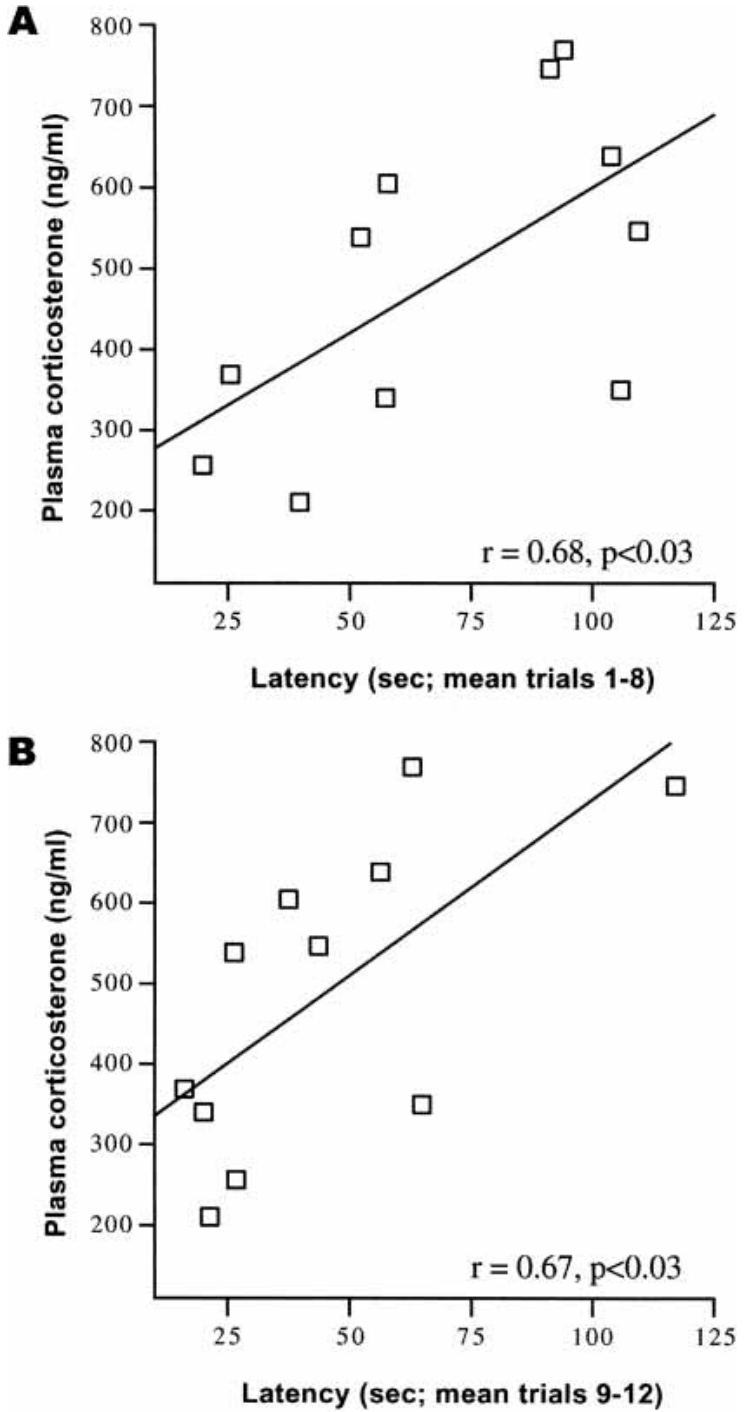

Figure 5 Positive correlations between plasma corticosterone levels exhibited by rats immediately after performance on the water maze on day 2 , and average latency to find the platform displayed by rats $(A)$ at training (trials $1-8$, day 1 ) and $(B)$ at relearning (trials $9-12$, day $2 ; n=11$; Experiment 3).

to correlate with the levels of the NR1 subunit of the NMDA receptor in the dendrites of CA3 hippocampal pyramidal cells (Adams et al. 2001). Animals with better spatial learning ability had higher NR1 levels than subjects with poorer scores on the task. This result complements our findings on the inverse correlation between learning abilities and activated dentate PSA expression, suggesting that these two mechanisms of plasticity (i.e., NMDA receptors and NCAM polysialylation) might be differentially deployed during information processing, in a manner that is dependent on the cognitive effort required. Moreover, available evidence suggests that these two systems could be mutually inhibitory (Bouzioukh et al. 2001a,b; Nacher et al. 2001, 2002). For example, in the adult dorsal vagal complex, NMDA receptor activation was shown to induce a rapid down-regulation of PSANCAM expression (Bouzioukh et al. 2001a).

It should be noted that water maze training-induced upregulation of PSA was found $12 \mathrm{~h}$ posttraining, regardless of whether a 60-min probe test was given (this study), or not (Murphy et al. 1996), just before decapitation, which strongly sup- ports the view that PSA modulation detected in our study was triggered by the training session. In fact, the brief period of swimming during the probe test could not account for PSA biosynthesis (Kiss and Rougon 1997). Therefore, PSA modulation occurring several hours after training appears to be related to the consolidation of long-term memory (Murphy and Regan 1998). Nevertheless, further experiments are required to assess whether the differential performance observed at training is related to inherent neurobiological differences of individuals. Interestingly, a priori differences in hippocampal morphometry (BernasconiGuastalla et al. 1994; Schwegler and Crusio 1995), neurochemistry (Huber et al. 1997; McIntyre et al. 2003), and expression of the cell adhesion molecule L1 (Venero et al. 2004) have been related to differential performance in spatial learning tasks in adult rodents.

Furthermore, our findings indicate that the correlation found between water maze performance and PSA regulation is related, not only to performance in a single session, but to a pattern of spatial learning and memory abilities, and, moreover, learning-related glucocorticoid responsiveness. Performance in the massed training protocol was shown to be indicative of a consistent pattern of cognitive abilities, as it was highly related to performance of rats when subsequently confronted with other learning and memory challenges in the same maze. Correlation analyses indicated that the better the performance on day 1: (1) the higher the retention of the platform location in the probe test performed $24 \mathrm{~h}$ posttraining (however, note that only a trend toward a correlation was found in Experiment 1, when the probe test was performed $12 \mathrm{~h}$ posttraining); (2) the better the performance on a relearning session of the task; and (3) the better the ability to learn a new spatial location in the reversal learning task.

In addition, we found that circulating corticosterone levels after the relearning task on day 2 were positively correlated with the latency required by rats to find the platform, both on day 1 and day 2 (Experiment 3). Although this result could be interpreted as a consequence of differences in swim duration between fast and slow learners, the fact that such a correlation is not found in Experiment 4, in animals submitted to a reversal learning session, brings into question such an interpretation. In our view, supported by the fact that blood samples were taken in all animals at the same time after the beginning of the first trial on day 2 , such a correlation might reflect a differential stress response associated with learning abilities. The fact that no correlation was observed in Experiment 4 is most probably due to the complexity of the demands involved in the reversal learning task, where performance of animals is not only dependent on learning abilities, but also, among other factors, on the strength of the memory for the previous platform location. Furthermore, the lack of correlation between behavioral performance and corticosterone levels after the probe test is most probably due to the very short period when hormone levels were assessed after initial performance, which is not enough time to trigger the hypothalamus-pituitary-adrenocortical cascade to detect any changes in corticosterone release from the adrenals. Therefore, the correlation observed between the learning rate of a stable location of the platform and posttraining corticosterone levels in Experiment 3 further supports the view that our massed water maze training protocol allows characterization of a relatively stable pattern of individual differences in cognitive abilities and endocrine reactivity. Given the reported role of glucocorticoid hormones in the consolidation of hippocampus-dependent tasks (Oitzl and de Kloet 1992; Roozendaal and McGaugh 1997; Sandi et al. 1997) and the ability of glucocorticoids to regulate hippocampal NCAM polysialylation (Rodriguez et al. 1998), further studies will be addressed to explore the possible relationship between vari- 
ability in glucocorticoid responsiveness and individual differences in neural systems for learning and memory.

Thus, our findings suggest that the learning-related neural circuits of fast learners are better suited to solving the water maze task than those of slow learners, the latter requiring structural reorganization to form memory as opposed to the relatively economic mechanism of altering synaptic efficacy used by the former. Further studies will need to address whether differential, learning-associated activation of PSA observed following other learning paradigms is also reflective of inherent individual differences in learning ability. Moreover, our hypothesis that PSArelated synaptic remodeling is mobilized for memory formation in particularly challenging circumstances predicts that a sufficiently difficult task will see activation of hippocampal PSA expression and synaptic reorganization in all animals.

\section{MATERIALS AND METHODS}

\section{Animals}

Male Wistar rats (Harlan Iberica, Spain), weighing 150-175 g on arrival, were housed in groups of three per cage, under controlled conditions of temperature $\left(22^{\circ} \pm 2^{\circ} \mathrm{C}\right)$ and light $(12: 12$ lightdark cycle; lights on at 7 a.m.), and had free access to food and water in a colony room. At age $11 \mathrm{wk}$, they were handled daily for $3 \mathrm{~d}$ for habituation to the experimental manipulation. Experiments started following the third day of handling, when rats were postnatal day 80 . Behavioral experiments were always conducted between 09:30 and 14:30 h. Animal care procedures were conducted in accordance with the guidelines set by the European Community Council Directives (86/609/EEC).

\section{Water Maze Learning}

The Morris water maze was a black circular pool ( $2 \mathrm{~m}$ diameter, $45 \mathrm{~cm}$ high) filled with water $\left(30 \mathrm{~cm}\right.$ depth) at $25^{\circ} \mathrm{C}$. The pool was divided into four quadrants of equal size. An invisible escape platform (11 cm diameter) was placed in the middle of one of the quadrants $(1.5 \mathrm{~cm}$ below the water surface) equidistant from the side wall and middle of the pool. The behavior of the animal (latency, distance, swim speed, and thigmotactic swimming) was monitored by a video camera, mounted in the ceiling above the center of the pool, and a computerized tracking system (Ethovision 1.9; Noldus IT). Thigmotactic swimming is defined as the behavior that rats display when swimming close to the walls of the water maze. For its measurement, the maze was divided into two circles, and the time spent in the outer ring of the pool (18 cm wide) was designated as "thigmotactic swimming" (Gass et al. 1998).

Four different starting positions were equally spaced around the perimeter of the pool. The first training session always consisted of a massed training protocol to ensure a high acquisition level within a short period of time. This session consisted of eight trials, which were started from one of the four start positions in the same random sequence for each rat. A trial began by placing the rat into the water facing the wall of the pool at one of the starting points. If the rat failed to escape within $120 \mathrm{sec}$, it was guided to the platform by the experimenter. Once the rat reached the platform, it was allowed to stay there for $30 \mathrm{sec}$ and was then placed in a holding cage for an intertrial period of $30 \mathrm{sec}$. After the last trial, the rats were dried off by placing them in a waiting cage for $30 \mathrm{~min}$, in a room heated to $30^{\circ} \mathrm{C}$. In this cage there was always at least one companion rat to avoid any isolation stress. Subsequently, they were returned to their home cages.

Different behavioral experiments were performed. The first one (Experiment 1) was carried out to evaluate PSA-NCAM levels $12 \mathrm{~h}$ posttraining. Rats $(n=12)$ were tested $12 \mathrm{~h}$ after training for their retention of spatial orientation by giving them a 60 -sec free swim trial without a platform (probe test), and then decapitated. The percent time spent in the quadrant ("target" quadrant) in which the platform was previously located was assessed. Further experiments explored the pattern of behavioral and endocrine responses exhibited by rats once they had been characterized according to their spatial learning abilities in the first massed training session. Thus, $24 \mathrm{~h}$ after the massed training procedure, rats were submitted to (1) a probe test (Experiment 2; $n=12$ ); (2) a four-trials relearning session, with the platform located in the same place as on the previous day (Experiment $3 ; n=11$ ); or (3) a four-trials reversal learning session, with the platform located in the quadrant opposite to the previous day (Experiment 4; $n=6$ ). For correlational analyses, the first trial of the reversal learning session (trial 9) was discarded, because it is the first time that rats are confronted with a new platform location, and, therefore, their performance does not reflect memory for the location of the platform. A control group $(n=9)$ consisted of undisturbed rats of the same age that were sacrificed at the same time as trained rats.

\section{Determination of Plasma Corticosterone Levels}

In all experiments, immediately after the last behavioral testing, rats were decapitated and their trunk blood was collected for subsequent assessment of plasma corticosterone levels. Blood samples were centrifuged (3000 rpm for $20 \mathrm{~min}$ at $4^{\circ} \mathrm{C}$ ), and the obtained plasma was stored at $-35^{\circ} \mathrm{C}$. Corticosterone was measured using a radioimmunoassay (RIA) kit according to the instructions supplied by the manufacturer (Coat-A-Count, Diagnostics Products Corporation). The intra-assay variability of the RIA ranged between $3.2 \%$ and $4.7 \%$. Its sensitivity (minimal detectable dose) was $\sim 5.7 \mathrm{ng} / \mathrm{mL}$.

\section{Quantification of Dentate Hippocampal Polysialylated Neurons}

In Experiment 1, immediately after decapitation, the brains were removed and immediately frozen in dry ice-cooled $n$-hexane. Subsequently, the brains were coded and stored at $-80^{\circ} \mathrm{C}$ until required for further processing. PSA immunocytochemistry was used to detect hippocampal polysialylated neurons using techniques described previously (Fox et al. 1995). In brief, cryostatcut horizontal sections of $12 \mu \mathrm{m}$ were cut at $-5.6 \mathrm{~mm}$ with respect to bregma and fixed in $70 \%$ ethanol $\left(\mathrm{v} / \mathrm{v} \mathrm{H}_{2} \mathrm{O}\right)$ and incubated overnight with anti-PSA ascitic fluid (generous gift of G. Rougon, CNRS, Marseille, France) diluted 1:500 with phosphatebuffered saline (PBS). The sections were exposed for $3 \mathrm{~h}$ to fluorescein-conjugated goat anti-mouse IgM (Calbiochem) diluted 1: 100 in PBS and mounted in Citifluor (Agar), a fluorescenceenhancing medium. Nuclei were fluorescently counterstained by a brief $(60 \mathrm{sec})$ exposure to propidium iodide $(40 \mathrm{ng} / \mathrm{mL} \mathrm{PBS}$; Sigma Chemical Co.) to facilitate counting.

For the purposes of quantification, a PSA-immunopositive neuron was defined as a propidium-iodide-stained nucleus with a partial or complete somal membrane immunopositive for PSA. The total number of PSA-immunopositive neurons at the granule cell layer and hilus border was counted in seven alternate $12-\mu \mathrm{m}$ sections commencing $-5.6 \mathrm{~mm}$ from bregma thereby precluding double counting of the 5-10 $\mu \mathrm{m}$ perikarya. Cell counts were divided by the total area of the granule cell layer and multiplied by the average granular cell layer area, which was $0.15 \pm 0.01 \mathrm{~mm}^{2}$ at this level. The mean \pm SEM value was calculated, and the results were expressed as number of PSA-positive cells per unit area. Area measurements were performed using a Quantimet 500 Image Analysis System. Immunofluorescence was specific as it was eliminated by omission of either the primary or secondary antibody; by preabsorbing anti-PSA with colominic acid (1 mg/ $\mathrm{mL}$ ), which contains $\alpha 2,8$-linked homopolymers of sialic acid; or by prior incubation of the sections with $0.3 \%$ endoneuraminidase-N (v/v PBS; Fox et al. 1995; Murphy et al. 1996). Measurements were done by an experimenter blind to the treatment received by the animals from which sections were under analysis.

In addition to the current experiment, we also re-evaluated results obtained in our previous study (Murphy et al. 1996) that looked at PSA-positive cell frequency at increasing times (0-24 h) following a five-trial water maze training session. In particular, 
this analysis assessed correlation between learning performance, taken as the average latency to reach the platform across all five trials, and PSA-immunopositive infragranular neurons at times when no training-associated regulation of PSA was evident.

\section{Statistics}

All results were expressed as mean \pm SEM and analyzed by analysis of variance (ANOVA) or Student's t-tests, as appropriate. Pearson correlational analyses were performed to evaluate possible associations between the behavioral data and the morphological or the endocrine parameters obtained in the water-maze-trained animals. In all cases, $p<0.05$ was accepted to indicate significance.

\section{ACKNOWLEDGMENTS}

This work was supported by grants from the DGES (PM99-027, Spain) and the EU 5th Framework Programme (QLRT-02187). The authors thank Cesar Venero for valuable help.

The publication costs of this article were defrayed in part by payment of page charges. This article must therefore be hereby marked "advertisement" in accordance with 18 USC section 1734 solely to indicate this fact.

\section{REFERENCES}

Abrous, D.N., Montaron, M.F., Petry, K.G., Rougon, G., Darnaudery, M., Le Moal, M., and Mayo, W. 1997. Decrease in highly polysialylated neuronal cell adhesion molecules and in spatial learning during ageing are not correlated. Brain Res. 744: 285-292.

Adams, M.M., Smith, T.D., Moga, D., Gallagher, M., Wang, Y., Wolfe, B.B., Rapp, P.R., and Morrison, J.H. 2001. Hippocampal dependent learning ability correlates with $N$-methyl-D-aspartate (NMDA) receptor levels in CA3 neurons of young and aged rats. J. Comp. Neurol. 432: 230-243.

Banasr, M., Hery, M., Brezun, J.M., and Daszuta, A. 2001. Serotonin mediates oestrogen stimulation of cell proliferation in the adult dentate gyrus. Eur. J. Neurosci. 14: 1417-1424.

Becker, C.G., Artola, A., Gerardy-Schahn, R., Becker, T., Welzl, H., and Schachner, M. 1996. The polysialic acid modification of the neural cell adhesion molecule is involved in spatial learning and hippocampal long-term potentiation. J. Neurosci. Res. 45: 143-152.

Bernasconi-Guastalla, S., Wolfer, D.P., and Lipp, H.P. 1994. Hippocampal mossy fibers and swimming navigation in mice: Correlations with size and left-right asymmetries. Hippocampus 4: $53-63$.

Bouzioukh, F., Tell, F., Jean, A., and Rougon, G. 2001a. NMDA recepto and nitric oxide synthase activation regulate polysialylated neural cell adhesion molecule expression in adult brainstem synapses. $J$. Neurosci. 21: 4721-4730.

Bouzioukh, F., Tell, F., Rougon, G., and Jean, A. 2001b. Dual effects of NMDA receptor activation on polysialylated neural cell adhesion molecule expression during brainstem postnatal development. Eur. J. Neurosci. 14: 1194-1202.

Cazalis, F., Valabrègue, R., Pélégrini-Isaac, M., Asloun, S., Robbins, T.W., and Granon, S. 2003. Individual differences in prefrontal cortical activation on the Tower of London planning task: Implication for effortful processing. Eur. J. Neurosci. 17: 2219-2225.

Doyle, E., Bell, R., and Regan, C.M. 1992. Hippocampal NCAM180 transiently increases sialylation during the acquisition and consolidation of a passive avoidance response in the adult rat. $J$. Neurosci. Res. 31: 513-523.

Drapeau, E., Mayo, W., Aurousseau, C., Le Moal, M., Piazza, P.V., and Abrous, D.N. 2003. Spatial memory performances of aged rats in the water maze predict levels of hippocampal neurogenesis. Proc. Natl. Acad. Sci. 100: 14385-14390.

Durbec, P. and Cremer, H. 2001. Revisiting the function of PSA-NCAM in the nervous system. Mol. Neurobiol. 24: 53-64.

Eyre, M.D., Richter-Levin, G., Avital, A., and Stewart, M.G. 2003. Morphological changes in hippocampal dentate gyrus synapses following spatial learning in rats are transient. Eur. J. Neurosci. 17: $1973-1980$.

Foley, A.G., Hedigan, K., Roullet, P., Sara, S.J., Murphy, K.J., and Regan C.M. 2003. Consolidation of odour-reward associative memory involves neural cell adhesion molecule polysialylation-mediated synaptic plasticity within the rodent hippocampus. J. Neurosci. Res. 74: $570-576$.

Fox, G.B., O'Connell, A.W., Murphy, K.J., and Regan, C.M. 1995. Memory consolidation induces a transient and time-dependent increase in the frequency of neural cell adhesion molecule polysialylated cells in the adult rat hippocampus. J. Neurochem. 65: $2796-2799$

Gass, P., Wolfer, D.P., Balschun, D., Rudolph, D., Frey, U., Lipp, H.P., and Schütz. 1998. Deficits in memory tasks of mice with CREB mutations depend on gene dosage. Learn. Mem. 5: 274-288.

Habib, R., McIntosh, A.R., and Tulving, E. 2000. Individual differences in the functional neuroanatomy of verbal discrimination learning revealed by positron emission tomography. Acta Psychol.

105: $141-157$.

Huber, G., Bailly, Y., Martin, J.R., Mariani, J., and Brugg, B. 1997. Synaptic $\beta$-amyloid precursor proteins increase with learning capacity in rats. Neuroscience 80: $313-320$.

James, W. 1890. The principles of psychology. Dover Publications, New York.

Kelly, M.P. and Deadwyler, S.A. 2003. Experience-dependent regulation of the immediate-early gene Arc differs across brain regions. $J$. Neurosci. 23: 6443-6451.

Kiss, J.Z. 1998. A role of adhesion molecules in neuroglial plasticity. Mol. Cell. Endocrinol. 140: 89-94.

Kiss, J.Z. and Muller, D. 2001. Contribution of the neural cell adhesion molecule to neuronal and synaptic plasticity. Rev. Neurosci. 12: $297-310$.

Kiss, J.Z. and Rougon, G. 1997. Cell biology of polysialic acid. Curr. Opin. Neurobiol. 7: 640-646.

McIntyre, C.K., Marriot, L.K., and Gold, P.E. 2003. Patterns of brain acetylcholine release predict individual differences in preferred learning strategies in rats. Neurobiol. Learn. Mem. 79: 177-183.

McNaughton, N. 1997. Cognitive dysfunction resulting from hippocampal hyperactivity-A possible cause of anxiety disorder? Pharmacol. Biochem. Behav. 56: 603-611.

Merino, J.J., Cordero, M.I., and Sandi, C. 2000. Regulation of hippocampal cell adhesion molecules NCAM and L1 by contextual fear conditioning is dependent upon time and stressor intensity. Eur. I. Neurosci. 12: 3283-3290.

Morris, R.G.M., Garrud, P., Rowlins, J.N.P., and O'Keefe, J. 1982. Place navigation impaired in rats with hippocampal lesions. Nature 297: 681-683.

Muller, D., Wang, C., Skibo, G., Toni, N., Cremer, H., Calaora, V., Rougon, G., and Kiss, J.Z. 1996. PSA-NCAM is required for activity-induced synaptic plasticity. Neuron 17: 413-422.

Murphy, K.J. and Regan, C.M. 1998. Contributions of cell adhesion molecules to altered synaptic weights during memory consolidation. Neurobiol. Learn. Mem. 70: 73-81.

Murphy, K.J., O'Connell, A.W., and Regan, C.M. 1996. Repetitive and transient increases in hippocampal neural cell adhesion molecule polysialylation state following multi-trial spatial training. $J$. Neurochem. 67: 1268-1274.

Nacher, J., Rosell, D.R., Alonso-Llosa, G., and McEwen, B.S. 2001. NMDA receptor antagonist treatment induces a long-lasting increase in the number of proliferating cells, PSA-NCAM-immunoreactive granule neurons and radial glia in the adult rat dentate gyrus. Eur. J. Neurosci. 13: 512-520.

Nacher, J., Alonso-Llosa, G., Rosell, D., and McEwen, B. 2002. PSA-NCAM expression in the piriform cortex of the adult rat. Modulation by NMDA receptor antagonist administration. Brain Res. 927: $111-121$.

Nguyen, P.V., Abel, T., Kandel, E.R., and Bourtchouladze, R. 2000. Strain-dependent differences in LTP and hippocampus-dependent memory in inbred mice. Learn. Mem. 7: 170-179.

Nothias, F., Vernier, P., von Boxberg, Y., Mirman, S., and Vincent, J.D. 1997. Modulation of NCAM polysialylation is associated with morphofunctional modifications in the hypothalamo-neurohypophysial system during lactation. Eur. J. Neurosci. 9: 1553-1565.

Oitzl, M.S. and de Kloet, E.R. 1992. Selective corticosteroid antagonists modulate specific aspects of spatial orientation learning. Behav. Neurosci. 106: 62-71.

O’Malley, A., O'Connell, C., Murphy, K.J., and Regan, C.M. 2000. Transient spine density increases in the mid-molecular layer of hippocampal dentate gyrus accompany consolidation of a spatial learning task in the rodent. Neuroscience 99: 229-232.

Riedel, G., Micheau, J., Lam, A.G., Roloff, E., Martin, S.J., Bridge, H., Hoz, L., Poeschel, B., McCulloch, J., and Morris, R.G.M. 1999. Reversible neural inactivation reveals hippocampal participation in several memory processes. Nat. Neurosci. 2: 898-905.

Rodriguez, J.J., Montaron, M.F., Petry, K.G., Aurousseau, C., Marinelli, M., Premier, S., Rougon, G., Le Moal, M., and Abrous, D.N. 1998. Complex regulation of the expression of the polysialylated form of the neuronal cell adhesion molecule by glucocorticoids in the rat hippocampus. Eur. J. Neurosci. 10: 2994-3006.

Rønn, L.C., Berezin, V., and Bock, E. 2000. The neural cell adhesion 


\section{Sandi et al.}

molecule in synaptic plasticity and ageing. Int. J. Dev. Neurosci. 18: $193-199$.

Roozendaal, B. and McGaugh, J.L. 1997. Basolateral amygdala lesions block the memory-enhancing effect of glucocorticoid administration in the dorsal hippocampus of rats. Eur. J. Neurosci. 9: 76-83.

Rougon, G. 1993. Structure, metabolism and cell biology of polysialic acid. Eur. J. Cell. Biol. 61: 197-207.

Rowe, W.B., Spreekmeester, E., Meaney, M.J., Quirion, R., and Rochford, J. 1998. Reactivity to novelty in cognitively-impaired and cognitively-unimpaired aged rats and young rats. Neuroscience 83: 669-680.

Sadoul, R., Hirn, M., Deagostini, H., Rougon, G., and Goridis, C. 1983. Adult and embryonic mouse neural cell adhesion molecules have different binding properties. Nature 304: 347-349.

Sandi, C., Loscertales, M., and Guaza, C. 1997. Experience-dependent facilitating effect of corticosterone on spatial memory formation in the water maze. Eur. J. Neurosci. 9: 637-642.

Sandi, C., Davies, H.A., Cordero, M.I., Rodriguez, J.J., and Stewart, M.G. 2003a. Rapid reversal of stress induced loss of synapses in CA3 of rat hippocampus following water maze training. Eur. J. Neurosci. 17: 2447-2456.

Sandi, C., Merino, J.J., Cordero, M.I., Kruyt, N.D., Murphy, K.J., and Regan, C.M. 2003b. Modulation of hippocampal NCAM polysialylation and spatial memory consolidation by fear conditioning. Biol. Psychiatry 54: 599-607.

Schachner, M. 1997. Neural recognition molecules and synaptic plasticity. Curr. Opin. Cell. Biol. 9: 627-634.

Schulz, D., Huston, J.P., Jezek, K., Haas, H.L., Roth-Härer, A., Selbach O., and Luhmann, H.J. 2002. Water maze performance, exploratory activity, inhibitory avoidance and hippocampal plasticity in aged superior and inferior learners. Eur. J. Neurosci. 16: 2175-2185.

Schwegler, H. and Crusio, W.E. 1995. Correlations between radial-maze learning and structural variations of septum and hippocampus in rodents. Behav. Brain Res. 67: 29-41.
Seki, T. and Arai, Y. 1991. The persistent expression of a highly polysialylated NCAM in the dentate gyrus of the adult rat. Neurosci. Res. 12: 503-513.

. 1993. Highly polysialylated neural cell adhesion molecule NCAM-H is expressed by newly generated granule cells in the dentate gyrus of the adult rat. J. Neurosci. 13: 2351-2358.

. 1999. Different polysialic acid-neural cell adhesion molecule expression patterns in distinct types of mossy fiber boutons in the adult hippocampus. J. Comp. Neurol. 410: 115-125.

Seki, T. and Rutishauser, U. 1998. Removal of polysialic acid-neural cell adhesion molecule induces aberrant mossy fiber innervation and ectopic synaptogenesis in the hippocampus. J. Neurosci. 18: $3757-3766$

Simon, P., Dupuis, R., and Costentin, J. 1994. Thigmotaxis as an index of anxiety in mice. Influence of dopaminergic transmissions. Behav. Brain Res. 61: 59-64.

Smith, T.D., Adams, M.M., Gallagher, M., Morrison, J.H., and Rapp, P.R. 2000. Circuit-specific alterations in hippocampal synaptophysin immunoreactivity predict spatial learning impairment in aged rats. $J$. Neurosci. 20: 6587-6593.

Theodosis, D.T., Bonhomme, R., Vitiello, S., Rougon, G., and Poulain, D.A. 1999. Cell surface expression of polysialic acid on NCAM is a prerequisite for activity-dependent morphological neuronal and glial plasticity. J. Neurosci. 19: 10228-10236.

Tulving, E., Habib, R., Nyberg, L., Lepage, M., and McIntosh, A.R. 1999. PET correlations in and beyond medial temporal lobes. Hippocampus 9: $71-82$.

Venero, C., Tilling, T., Hermans-Borgmeyer, I., Herrero, A.I., Schachner, M., and Sandi, C. 2004. Water maze learning and forebrain mRNA expression of the neural cell adhesion molecule L1. J. Neurosci. Res. 75: $172-181$.

Received September 15, 2003; accepted in revised form March 2, 2004.

\section{Learning \& Memory}




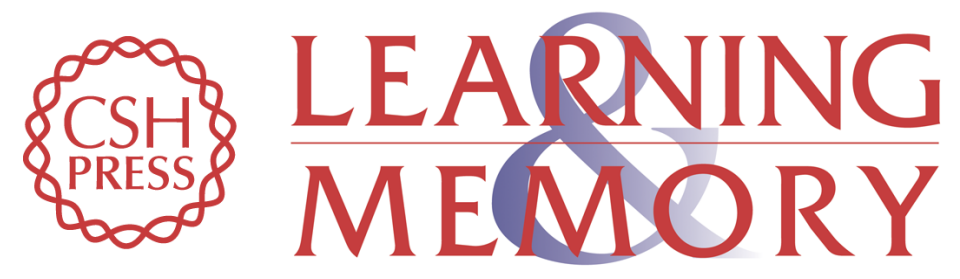

\section{Neurobiological and Endocrine Correlates of Individual Differences in Spatial Learning Ability}

Carmen Sandi, M. Isabel Cordero, José J. Merino, et al.

Learn. Mem. 2004, 11:

Access the most recent version at doi:10.1101/lm.73904

References This article cites 56 articles, 9 of which can be accessed free at:

http://learnmem.cshlp.org/content/11/3/244.full.html\#ref-list-1

License

Email Alerting Receive free email alerts when new articles cite this article - sign up in the box at the Service top right corner of the article or click here. 